\title{
Design and Simulation of Elephant Intrusion Detection System
}

\author{
Arya Singh S., Maneesha V. Ramesh and Divya P. \\ Amrita Center for Wireless Networks and Applications (AmritaWNA), Amrita School of Engineering, \\ Amritapuri, Amrita Vishwa Vidyapeetham, Amrita University, India
}

\begin{abstract}
Due to the increase in the population, human settlement is extending to forest border areas, causing conflicts between elephants and humans, leading to loss of life and property. Hence, large scale monitoring is required for real-time detection and warning of elephant intrusion into human settlements. Existing solutions using seismic waves, image processing, etc. are costly solutions for large scale monitoring. The project aims at developing an affordable solution for large-scale monitoring and early warning of elephant intrusion. In this work, architecture for elephant intrusion detection using wireless sensor networks is proposed and also a low cost sensor system has been designed and developed after selecting the appropriate sensors for monitoring. After analysing the existing signal processing methods, the most suitable technique for real-time detection of elephants is chosen and simulated. A two level detection system using microphones and IR sensors is used for monitoring the scenario and sound data is sent using FM transceivers to a PC.
\end{abstract}

Keywords: human-elephant conflicts, intrusion, IR sensors, FM transceivers, microphone.

\section{Introduction}

India is home to $60 \%$ of the elephant population in Asia. Two-thirds of this population has spread close to human habitation leading to human-elephant conflicts. Human-elephant conflicts is one of the main problems in India as it causes crop damage as well as human death and injuries. The main reason for the increasing man-elephant conflicts is due to the increased human population which extends their settlements into forest border areas. This migration leads to elephant's habitat fragmentation, and scarcity of food and water for the elephants. To avoid these conflicts, many detection methods exist, but they do not offer a proper protection system.

Elephants are large and heavy animals. Due to their size and the nature of their movement it is difficult to detect these animals. Many of the researchers have worked in this area but their works includes only detection and early warning systems for elephants. Most of the existing technologies for detection are based on seismic waves, image processing, etc. which are costly solutions for large scale monitoring of elephant and their intrusion into the human habitat. The main problem is that the sensors used for detecting these features are not much affordable for large area deployment. Also, for improving the accuracy and reliability of the detection, there is a need for a better signal processing technology.

There are many traditional techniques being used in the forest border areas to drive away the elephants once they intrude into the human settlement areas. They mainly use noise making techniques including firecrackers, pipe cannons, vehicle horns, shouts, rifle-shots, and banging on things such as drums, tins, etc. Sometimes, they create a fence strung with beehives made out of hollow logs and burn elephant dung along with chilli or any other material that will smoulder to create a heavy acrid smoke. However, as time passes, the elephants get used to these methods and develop the capability to overcome these traditional techniques. Hence a monitoring and detection system is needed to give warning ahead about the incoming elephants so that necessary protective measures can be adopted. 
Therefore, we are introducing a two level detection system to monitor the intrusion of elephants wherein a microphone is used in the first level and an IR LED is used in the second level of detection. This two level detection system helps to find whether the elephants are nearing the housing colony or not, thus disseminating warning at the appropriate times and thereby helping in reducing human-animal conflicts in forest areas.

\section{Related Work}

There exist so many methods to reduce the human-elephant conflicts. In [1] S. J. Sugumar et.al suggests an early warning intrusion detection system using geophone to reduce man-elephant conflicts in the forest border areas. The developed system automatically sends an early warning through SMS to the forest officials to take necessary precautions and actions. In [2] S. J. Sugumar et.al introduced an elephant image detection system (EIDS) as a solution to reduce human-elephant conflicts. In that, they proposed an idea of sending a GSM message to the forest officials when an elephant has been detected. J. Nirmal Prince et.al presented a system to reduce human-elephant conflicts, which detects and tracks elephants along the border of the forest using the vocal communications of elephants [3]. The experiment has two phases: one is to find the spectral magnitude and the highest pitch frequency of elephant's sound signal. As the second phase, an alert is sent to the forest officials notifying about the elephant intrusion. Rizki Dian Rahayani et.al proposed a system to detect the intrusion of elephants in human settlement [4]. The proposed system monitors the position of the elephants in human habitat using radio frequency module and it gives an early warning to the guards and people around. In [5] C. E. O'Connell-Rodwella et.al did their experiments using seismic and acoustic data, which was recorded simultaneously during period of vocalizations and locomotion. The data analyses were based on elephant's rumbles and foot stomps signal. They concluded from their experiments that the acoustic signals are traveling at $309 \mathrm{~m} / \mathrm{s}$ through air and the seismic signals at 248-264 m/s.

In other related research, Seung You Na et.al proposed a system for protection of orchard fruits from wild birds and animals in [6]. They proposed a method to protect orchard produce via ubiquitous sensor network devices and also proposed monitoring scheme to provide an early warning about possible intrusion and damage by wild animals and birds. In [7] Peter Christiansen et.al presented a system for reducing the death of animals in agricultural mowing operations. They presented an automated detection and classification of animals using thermal imaging.

All the studies mostly investigate the possibility of reducing human-elephant conflicts by developing an efficient single level elephant intrusion and alert dissemination system, but they did not consider the cost of sensors. Our major concern is about developing a real time multi-level detection system for large area monitoring with low cost sensors. The proposed work aims to model an affordable and efficient multi-level intrusion detection system for large area monitoring.

\section{System Architecture}

We propose the architecture of an affordable, and an efficient elephant intrusion detection system. This is a two level detection system for large area monitoring. The system mainly uses microphones and IR LEDs to sense the presence of elephants. The first level detection focuses on use a microphone that is $50 \mathrm{~m}$ away from the housing colony. It captures sound data, which is then sent through a FM transmitter, and the received signal is analysed using MATLAB. Thus we can detect the intrusion of an elephant within a radius of 50 meters from the human settlement using this first level sensor system. The second level uses IR sensors, which is $20 \mathrm{~m}$ away from the housing colony. If an elephant crosses the IR sensor, the breaking time for the IR beam is calculated to detect the presence of the elephant within the 20 meter distance from the housing colony. The overall architecture is shown in Fig. 1

\subsection{Sound Detection System}

Sound detection is the first level detection. The sound detection system has a microphone, an FM transmitter and an FM Receiver. Microphones capture the sound signal and send it through the FM transmitter. The FM receiver is usually connected to a PC or a laptop at the monitoring station. It receives that signals and transmit it serially to the PC. The received sound signal is compared with the sound of an 
elephant stored in the database using MATLAB tool. Finally the presence of the elephant is detected if the compared signals match.

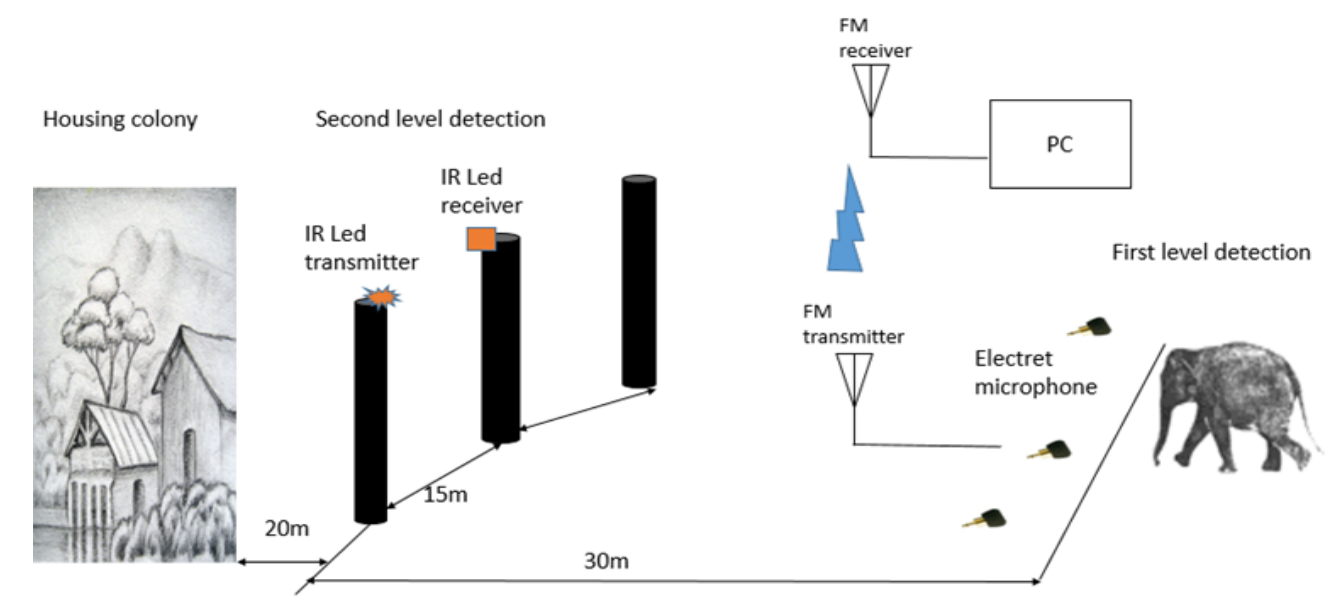

Fig. 1: Overall architecture.

The signal processing method used here is to capture the sound using a microphone. The captured signal is an analog continuous-time signal. The proposed algorithm compares the sound signal with existing data base. In this analysis, the recorded signal is filtered with the hamming filter with a window size of $20 \mathrm{~ms}$ and then modified. Thereafter, the FFT of the captured signal is taken to find the spectrum. To analyse the sound signal, Mel Frequency cepstrum analysis is used. It is a short-term power spectrum of the sound based on a linear cosine transform of a $\log$ power spectrum on a nonlinear Mel scale of frequency. Finally, the DCT of cepstrum co-efficient is analysed. SVM classifier is used to analyse these values with stored database, then to detect the elephant. The algorithm is explained in Fig. 2.

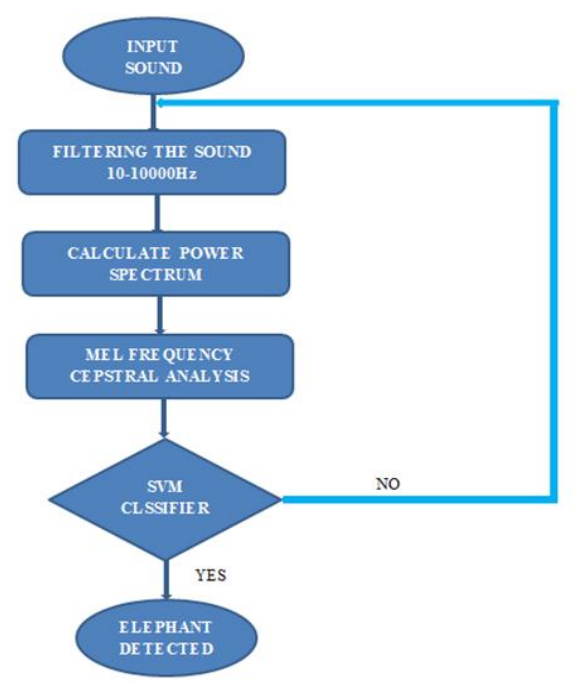

Fig. 2: Flow chart of sound detection algorithm.

\subsection{IR Crossing Detection System}

Second level of the system is IR detection. In that, we calculate the IR break time to detect the presence of the elephant. There are two circuits for IR detection, one is IR transmitter circuit and one IR is receiver circuit. This transmitter and receiver circuits have separation of $15 \mathrm{~m}$ distance at the maximum. The transmitter circuit has one timer and High watt IR LED, which generates pulses of about $38 \mathrm{KHz}$ on and off. The IR receiver receives a pulse train of $38 \mathrm{KHz}$. The receiver circuit has a TSOP and PIC. The output of TSOP is active low and is given as an input to the micro controller. The demodulated output signal can directly be decoded by a microprocessor.PIC calculates the IR break time with respect to the output of the IR receiver. A Led is connected to the PIC, if an elephant cross the IR fence Led starts to blink and it shows the intrusion of elephant. 
IR crossing detection algorithm is used to calculate the IR beam breaking time when an elephant move across IR sensor. The breaking time of IR beam depends upon the walking speed of elephants. We have analysed the IR beam breaking time for two different cases. In the first case, we considered the elephants walking in normal speed. IR beam breaking time of elephants walking in faster is considered as the second case. These analysed data are considered as the database for compare with the new sample data.

\section{Experiments and Results}

The hardware components used in the sound detection system mainly include a microphone for collecting the air acoustic signals, FM transmitter, FM receiver, IR led, battery to power the system and a laptop for processing the acquired data. The microphone used for acquiring data is omnidirectional and have sensitivity of $0 \mathrm{~dB}=1 \mathrm{~V} / \mathrm{Pa} 1 \mathrm{KHz}$, signal to noise ratio more than $58 \mathrm{~dB}$ and sensitivity reduction $3 \mathrm{~dB}$ at $1.5 \mathrm{~V}$. The detection frequency range is from nearly $10 \mathrm{~Hz}-16,000 \mathrm{~Hz}$. An FM transmitter is connected to the microphone. The range of transmission frequency of FM transmitter is $88-109 \mathrm{MHz}$. The captured data is sent from the FM Transmitter to the FM receiver. FM receiver transmits the data serially to the PC wherein the data obtained is analyzed using MATLAB tool.

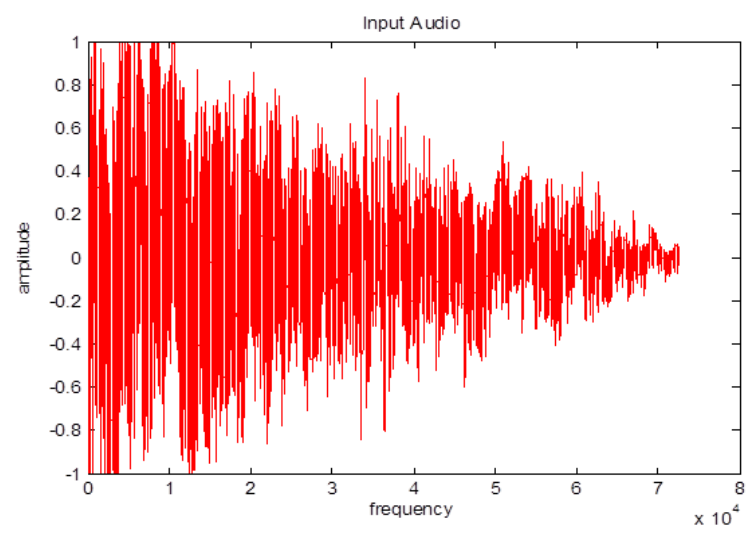

Fig. 3: Some jungle sound.

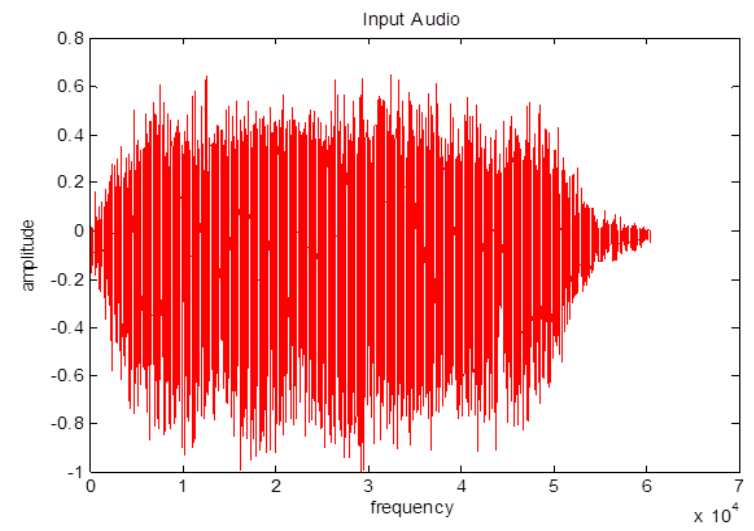

Fig. 5: Elephant sound.

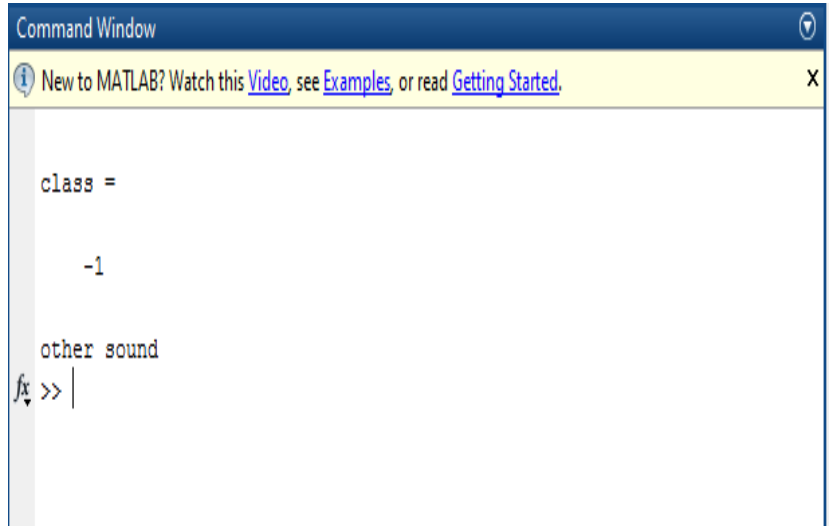

Fig. 4: Output of the detection algorithm.

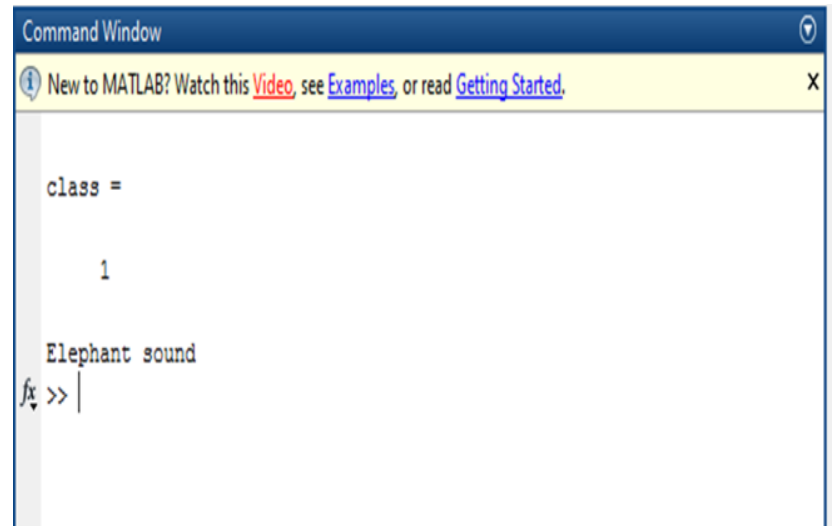

Fig. 6: Output of the detection algorithm.

The second level detection is IR detection, in which IR transmitter and receiver circuits are used. IR transmitter circuit has an IR transmitter LED with timer circuit for generating $38 \mathrm{kHz}$ square waves. The receiver circuit has a TSOP IR receiver to receive the $38 \mathrm{kHz}$ square pulses from the transmitter. The TSOP connected to a PIC micro controller, which count the time of IR beam break and analyze the data, and blinks a LED if elephant crosses the IR. The results obtained for the sound detection system are plotted in Fig: 3, 4, 5 , and 6 . The elephant's sound varies in frequency range between $5-10000 \mathrm{~Hz}$. Here we consider the sound signals of elephants and other jungle sounds in the above range. 


\section{Conclusion}

Taking into consideration the frequent incidents of human-elephant conflicts in the forest border areas, this work mainly aims at reducing elephant intrusion in human habitation area nearer to forest. This work proposes a two level detection system, which helps people and authority to take the protection measures when elephant is detected by the first level sensor system. The architecture of the low cost and simple two level system includes elephant intrusion detection system based on vocalization and IR beam breaking due to elephant presence. The first level is sound detection, in which the testing of real time vocalization signal detection using MATLAB tool is performed and in second level IR beam breaking time determines the presence of elephant. The simulation results of the vocalization signal and IR beam breaking details is also included in this report. The microphone configuration and the IR beam breaker were designed in a way suitable for this application. There are areas where wild elephants cause horrible damages and people and authority become helpless. The proposed idea gives the best solution to such situations by giving early warning to the authority and people.

\section{Acknowledgement}

We would like to express our gratitude for the guidance, support and motivation provided by Sri Mata Amritanandamayi Devi, the Chancellor, Amrita University, and also thank to our loving parents.

\section{References}

[1] S. J. Sugumar and R. Jayaparvathy., An early warning system for elephant intrusion along the forest border areas, Current Science 104.11, 2013.

[2] S. J. Sugumar and R. Jayaparvathy., An improved real time image detection system for elephant intrusion along the forest border areas. The Scientific World Journal 2014, 2014.

[3] J. N. Prince and S. J. Sugumar., Surveillance and tracking of elephants using vocal spectral information, International Journal of Research in En-gineering and Technology, 2014.

[4] A. G. Rahayani, Rizki Dian and A. U. Ariwibowo, Implementation of ra-dio frequency as elephant presence detector for the human elephant conflict prevention, Innovative Systems Designs and Engineering, 2014.

[5] e. a. Na, Seung You, Protection of orchard from wild animals and birds using usn facilities. Computer and Automation Engineering (ICCAE), 2010 the 2nd International Conference on, 2010.

[6] e. a. Christiansen, Peter, Automated detection and recognition of wildlife using thermal cameras, Sensors, 2014

[7] L. A. H. C. E. OConnell-Rodwella, B. T. Arnason, Seismic properties of asian elephant (elephas maximus) vocalizations and locomotion, article in the journal of the acoustical society of america,2001. 